Nephrologe 2009 • 4:487-487 DOI 10.1007/s11560-009-0364-y

(c) Springer Medizin Verlag 2009

\author{
H. Haller \\ Abteilung Nephrologie, Medizinische Hochschule Hannover
}

\title{
Niere und Hypertonie
}

Die vorliegende Ausgabe von „Der Nephrologe" beschäftigt sich mit dem Thema Hypertonie. Wir haben versucht, auf der einen Seite die neuen Entwicklungen auf dem Gebiet der Hypertonie darzustellen und anderseits wichtige Themen bei Diagnostik und Therapie aufzugreifen. Der erste Beitrag gibt einen Überblick über die Pathogenese der Hypertonie. Genauer handelt es sich um eine neue Theorie, welche den Salz-Wasser-Haushalt der eng mit der Pathogenese der Hypertonie verbunden ist - neu erläutert. Jens Tietze hat ausgehend von Untersuchungen zur Ablagerung von Kochsalz gezeigt, dass unsere landläufige Vorstellung von Volumen und Kochsalzregulation modifiziert und um die Ablagerung von Natrium im Interstitium erweitert werden muss. Diese Hypothese hat in den letzten Monaten viel Diskussion im Fachbereich ausgelöst, und wir wollen sie den Lesern von „Der Nephrologe" nicht vorenthalten.

Der zweite Beitrag geht auf das wichtige Thema "Demenz und Hypertonie“ ein. Während ursprünglich vor allem der akute Schlaganfall in der Hypertensiologie im Vordergrund stand, ist in den letzten Jahren deutlich geworden, dass auch chronische zerebrale Veränderungen durch Hypertonie begünstigt werden. Bei der zunehmenden Problematik von Demenz und degenerativen chronischen zerebralen Erkrankungen ist dies ein wichtiges Thema. Jochen Schrader geht sowohl auf die komplexe Diagnostik der funktionellen Störungen als auch auf die bildgebenden Verfahren ein und gibt einen Überblick der derzeitigen existierenden therapeutischen Vorstellungen.

Auch der dritte Beitrag behandelt ein wichtiges Thema. In den letzten Jahren ist es zu einer dramatischen Zunahme an interventionellen Eingriffen zur Behandlung von renovaskulärer Hypertonie gekommen. Bessere technische Möglichkeiten und die Zunahme von invasiven diagnostischen Maßnahmen in der Angiologie haben dazu geführt, dass die Nierenarterienstenose nicht nur häufiger diagnostiziert, sondern auch häufiger therapeutisch angegangen wird. Diese starke Zunahme von Intervention steht evidenzbasiert auf wackligen Beinen. Jörg Radermacher fasst den Stand der Diskussion um die Diagnostik und die Therapie der Nierenarterienstenosen nochmals kritisch zusammen.

Im letzten Beitrag habe ich versucht, Neues aus der Hypertonie des letzten Jahres zusammenzutragen und übersichtlich darzustellen. Fortschritte auf dem Gebiet der Genetik, Neues aus der Diagnostik und ein kurzer Überblick der wichtigsten Therapiestudien der letzten Jahre stellen den derzeitigen Stand in der Klinik und Forschung der Hypertonie dar.

Ich wünsche Ihnen beim Lesen des Heftes viel Freude und hoffe, Ihnen Neues und Interessantes mitteilen zu können.

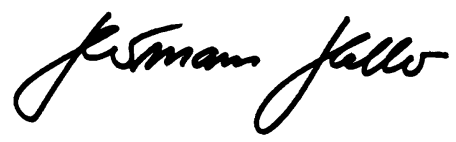

H. Haller

\section{Korrespondenzadresse Prof. Dr. H. Haller}

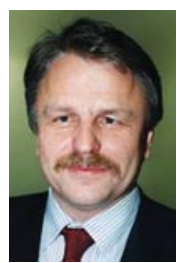

Abteilung Nephrologie, Medizinische Hochschule Hannover Carl-Neuberg-Str. 1, 30625 Hannover Haller.Hermann@ MH-Hannover.de 